\title{
REGULARLY VARYING SEQUENCES
}

\author{
J. GALAMBOS AND E. SENETA ${ }^{1}$
}

\begin{abstract}
A simple necessary and sufficient condition is developed for a sequence $\{\theta(n)\}, n=0,1,2, \cdots$, of positive terms, to satisfy $\theta(n)=R(n), n \geqq 0$, where $R(\cdot)$ is a regularly varying function on $[0, \infty)$. The condition (2.1), below, leads to a Karamatatype exponential representation for $\theta(n)$. Various associated difficulties are also discussed. (The results are of relevance in connection with limit theorems in various branches of probability theory.)
\end{abstract}

1. Introduction. A function $R(\cdot)$, defined, finite, positive and measurable on $[A, \infty)$ for some $A \geqq 0$, is said to be regularly varying if for each $\lambda>0$

$$
\lim _{x \rightarrow \infty} \frac{R(\lambda x)}{R(x)}=\phi(\lambda)
$$

where $0<\phi(\lambda)<\infty$. (In actual fact a weaker definition can be used, for the assumption that this positive finite limit property obtains for all $\lambda$ in a subset of positive measure of $(0, \infty)$ implies that it obtains for all $\lambda \in(0, \infty)$.) Since $\phi(\lambda)$ is a positive measurable solution of the functional equation

$$
\phi(u v)=\phi(u) \phi(v), \quad u, v>0,
$$

it is well known that $\phi(\lambda)=\lambda^{\rho}$ for some finite $\rho$, and so we can write $R(x)=x^{\rho} L(x)$ where

$$
\lim _{x \rightarrow \infty} \frac{L(\lambda x)}{L(x)}=1, \text { for each } \lambda>0 ;
$$

such a regularly varying function, for which the index $\rho$ of regular variation is zero, is called slowly varying.

Received by the editors January 30, 1973.

AMS (MOS) subject classifications (1970). Primary 26A12, 40A25; Secondary 10K30, 60F05.

Key words and phrases. Regularly varying functions, regularly varying sequences, imbedding sequences in functions, exponential representations of sequences and functions.

${ }^{1}$ Partial support by the Office of Naval Research on contract N00014-67-A-0151-0017 awarded to the Department of Statistics, Princeton University, is gratefully acknowledged.

(C) American Mathematical Society 1973 
The two most important properties of regularly varying functions (from which others are easily deducible) are:

(i) The convergence in (1.1) (or, equivalently, (1.3)) is uniform for $\lambda$ in any fixed interval $[a, b], 0<a<b<\infty$.

(ii) For some $B \geqq A$, a slowly varying function $L$ has representation

$$
L(x)=\exp \left\{\eta(x)+\int_{B}^{x} \frac{\varepsilon(t)}{t} d t\right\}, \quad x \geqq B,
$$

where $\eta(x) \rightarrow c(|c|<\infty)$ as $x \rightarrow \infty$ and $\varepsilon(t) \rightarrow 0$ as $t \rightarrow \infty$, both being measurable and bounded. Conversely, any function $L$ having representation (1.4) is clearly slowly varying.

The systematic development of the notion of a regularly varying function, of great importance in probability theory, is due, for continuous functions, to Karamata ([1930], [1933]), and in the above setting to various later authors. A sketch of the basic history and theory is given in $\S \S 1$ and 4 of the recent paper of Bojanić and Seneta [1971]. We pause to note only the result of de Bruijn [1959, $\$ 4$ ] that in (1.4), $\varepsilon(t)$ may be taken as continuous (the less desirable properties of $L$ being perpetuated by $\eta(x))$. This last remark enables us to deduce that as $x \rightarrow \infty$

$$
R(x)=x^{\rho} L(x) \sim x^{\rho} L_{1}(x)=R_{1}(x)
$$

where $\left(R_{1} x\right)=x^{\rho} \exp \left\{c+\int_{B}^{x} \varepsilon(t) / t d t\right\}, x \geqq B$, is a continuously differentiable regularly varying function such that

$$
x R_{1}^{\prime}(x) / R_{1}(x) \rightarrow \rho
$$

as $x \rightarrow \infty$, since

$$
x R_{1}^{\prime}(x) / R_{1}(x)=\rho+\varepsilon(x), \quad x \geqq B .
$$

Conversely, any function $R_{1}$ satisfying (1.5) is regularly varying (with index $\rho$ ), as can be seen by defining $\varepsilon(x)$ from (1.6) and integrating for $R_{1}$, to obtain the required representation.

More recently, a problem of the following genre has occurred in several probabilistic contexts. Given a sequence $\{\theta(n)\}, n=0,1,2, \cdots$, of positive numbers, when is it possible to imbed it in a regularly varying function? In other words, when is it possible to find a regularly varying function $R(x)$ such that $R(n)=\theta(n)$ ? If is it possible, then it follows, for example, from either property (i) or (ii) of regularly varying functions, that

$$
\theta(n+1) / \theta(n) \rightarrow 1
$$

as $n \rightarrow \infty$. As examples of results obtained so far, we mention that of 
de Haan [1970, pp. 6-8], who shows that the imbedding is possible if (a) $\{\theta(n)\}$ is monotone, and (b) $\theta(n m) / \theta(n) \rightarrow m^{\rho}$ for all positive $m$ as $n \rightarrow \infty$, where $\rho$ is finite, and that of R. S. Slack, which asserts that in (b), $m^{\rho}$ may be replaced by $\phi(m), 0<\phi(m)<\infty$, if $(1.7)$ is imposed as an additional hypothesis, with the same conclusion. ${ }^{2}$

This type of problem, concerning regular behavior of sequences, was studied prior to the papers of Karamata mentioned above. The reader may want to consult the works of Schmidt [1925] and Schur [1930] in this regard.

There is some difficulty in attempting the obvious approach to the sequence problem along the lines of the elegant definition (1.1). Thus, it is possible to construct a sequence of positive numbers $\{\theta(n)\}$ satisfying simultaneously (for positive integer $k$ ), as $n \rightarrow \infty$,

$$
\theta(n k) / \theta(n) \rightarrow 1, \quad \theta(n+1) / \theta(n) \rightarrow 1,
$$

so that the requirement (1.7) is broken.

To carry out such a construction, let $\omega(n)$ denote the number of prime divisors of $n$. Let $\theta(n)=\omega(n)+(\log \log n)^{1 / 2}, n \geqq 2$. It is known (Kubilius [1964, p. 39]) that there exists a subsequence $p_{j_{1}}, p_{j_{2}}, \cdots$ of the primes such that $\omega\left(p_{j_{n}}-1\right) \sim \log \log p_{j_{n}}$ as $n \rightarrow \infty$. If we consider the subsequence $\theta\left(p_{j_{n}}\right) / \theta\left(p_{j_{n}}-1\right)$ of the sequence $\theta(n+1) / \theta(n)$, we readily see that its limit is zero, since $\omega\left(p_{j_{n}}\right)=1$, so that $\theta(n+1) / \theta(n) \rightarrow 1$. If we consider $\theta^{*}(n)=$ $\omega(n)+\log \log n$ instead, we obtain that $\theta^{*}\left(p_{j_{n}}\right) / \theta^{*}\left(p_{j_{n}}-1\right) \rightarrow \frac{1}{2}$ as $n \rightarrow \infty$, also a satisfactory result. On the other hand, we have for integer $k \geqq 1$

$$
\theta(n k)=\omega(n k)+(\log (\log n+\log k))^{1 / 2},
$$

whence for large $n$,

$$
\begin{aligned}
& \omega(n)+(\log (\log n+\log k))^{1 / 2} \\
& \quad \leqq \theta(n k) \leqq \omega(n)+\omega(k)+(\log (\log n+\log k))^{1 / 2}
\end{aligned}
$$

from the definition $\omega$; and so $\theta(n k) / \theta(n) \rightarrow 1$, each integer $k \geqq 1$. (It may be proved similarly that $\theta^{*}(n k) / \theta^{*}(n) \rightarrow 1$.)

To conclude this section, it is necessary to mention that Ibragimov and Linnik [1971, p. 397] seem to cite, as an example of a sequence of positive terms such that $\theta(n k) / \theta(n) \rightarrow 1$ as $n \rightarrow \infty$, but $\theta(n+1) / \theta(n) \rightarrow 1$, the sequence given by $\theta(n)=\omega(n)+(\log n)^{1 / 2}$. Whereas it is easy to check that $\theta(n k) / \theta(n) \rightarrow 1$ for each positive integer $k$, the proposition regarding

${ }^{2}$ Mentioned in a letter to one of the authors from G. E. H. Reuter. Slack's result occurs in a branching process context, and his method is not known to us. Nevertheless, one of us has constructed a possibly different proof. 
$\theta(n+1) / \theta(n)$ appears to be a deeper one whose validity or otherwise is not known; but note that $\theta\left(p_{j_{n}}\right) / \theta\left(p_{j_{n}}-1\right) \rightarrow 1$ as $n \rightarrow \infty$.

2. Regularly varying sequences. We call a sequence $\{\theta(n)\}$ of positive terms regularly varying if there is a sequence of positive terms $\{\alpha(n)\}$ satisfying

$$
\begin{gathered}
\theta(n) \sim K \alpha(n), \quad K \text { a positive constant, } \\
n(1-\{\alpha(n-1) / \alpha(n)\}) \rightarrow \rho, \quad \rho \text { finite. }
\end{gathered}
$$

The number $\rho$ will be called the index of regular variation. The case $\rho=0$ may be called slowly varying. It is first necessary to note that there exist regularly varying sequences $\{\theta(n)\}$ which themselves do not satisfy the condition (2.1b) with $\alpha$ replaced by $\theta$ (just as not all regularly varying functions can satisfy (1.5), although $R(x) \sim R_{1}(x)$ always). For example, if we take $\theta(n)=1+(-1)^{n} / n, n \geqq 2$, then $\theta(n)$ is regularly varying with index 0 , since appropriate $\alpha(n)$ is given by $\alpha(n)=1$. However

$$
\begin{array}{rlrl}
n(1-\{\theta(n-1) / \theta(n)\}) & \rightarrow-2, & & \rightarrow \infty, n \text { odd }, \\
& \rightarrow 2, & n \rightarrow \infty, n \text { even. }
\end{array}
$$

We shall say that a sequence of positive terms $\{\theta(n)\}, n=0,1,2, \cdots$, is imbeddable in a regularly varying function $R$ on $[0, \infty)$ if $R(n)=\theta(n)$, $n \geqq 0$.

LEMma. If $\{\theta(n)\}, n=0,1,2, \cdots$, is a regularly varying sequence of index $\rho$, then it has representation

$$
\theta(n)=n^{\rho} a(n) \exp \left\{\sum_{j=1}^{n} \frac{\varepsilon(j)}{j}\right\}, \quad n \geqq 1,
$$

where, as $n \rightarrow \infty, a(n) \rightarrow$ positive limit, $\varepsilon(n) \rightarrow 0$.

Proof. Since $\theta(n) \sim K \alpha(n)$, we may assume without loss of generality that $1-\alpha(m-1) / \alpha(m) \equiv \rho / m+\varepsilon(m) / m, m \geqq 1$, is less than unity in modulus for all $m \geqq 1$, by changing the first few terms of $\{\alpha(n)\}$. Since for $|x|<1$, $-\log (1-x)=\sum_{k=1}^{\infty} x^{k} / k$, we obtain

$$
-\log \left\{\frac{\alpha(m-1)}{\alpha(m)}\right\}-\sum_{k=2}^{\infty} \frac{1}{k}\left\{1-\frac{\alpha(m-1)}{\alpha(m)}\right\}^{k}=\frac{\rho}{m}+\frac{\varepsilon(m)}{m} .
$$

Summing over $m$ from 1 to $n$,

$$
\log \alpha(n)-\log \alpha(0)-\sum_{m=1}^{n} \sum_{k=2}^{\infty} \frac{1}{k}\left\{1-\frac{\alpha(m-1)}{\alpha(m)}\right\}^{k}=\rho \sum_{m=1}^{n} \frac{1}{m}+\sum_{m=1}^{n} \frac{\varepsilon(m)}{m} .
$$


Now it is well known that $\sum_{m=1}^{n} m^{-1}-\log n=\gamma+o(1)$ as $n \rightarrow \infty$, where $\gamma$ is a positive constant. Further since for each integer $k \geqq 2$, from (2.1b),

$$
\left|\{1-\alpha(m-1) / \alpha(m)\}^{k}\right|<\left(|\rho|+\delta_{1}\right)^{k} / m^{k}
$$

for arbitrary fixed positive $\delta_{1}$, and positive integer $m$ sufficiently large (independently of $k$ ), we have the upper bound

$$
=\left(\left(|\rho|+\delta_{1}\right) / m^{1 / 4}\right)^{k} / m^{3 k / 4} \leqq \delta_{2}^{k} / m^{3 / 2}
$$

for $k \geqq 2$ and with $0<\delta_{2}<1$, for $m$ large (independent of $k$ ). Thus we obtain that the series $\sum_{m=1}^{\infty} \sum_{k=2}^{\infty} k^{-1}\{1-\alpha(m-1) / \alpha(m)\}^{k}$ is (absolutely) convergent. Hence it follows that

$$
\alpha(n)=n^{\rho} a(n) \exp \left\{\sum_{j=1}^{n} \frac{\varepsilon(j)}{j}\right\}
$$

where, as $n \rightarrow \infty, a(n) \rightarrow$ positive limit, $\varepsilon(n) \rightarrow 0$. Since $K(n) \equiv \theta(n) / \alpha(n) \rightarrow$ pos. const. by $(2.1 \mathrm{a})$, it follows that $\theta(n)$ has the same kind of representation, as required.

THEOREM. A sequence of positive terms $\{\theta(n)\}, n \geqq 0$, is imbeddable in a regularly varying function $R$ on $[0, \infty)$ if and only if the sequence is also regularly varying.

Proof. Sufficiency. If $\{\theta(n)\}$ is regularly varying with index $\rho$, we have representation (2.2) available for $\theta(n), n \geqq 1$. Put (where $[u]$ denotes the integer part of $u$ )

$$
\begin{aligned}
& R(0)=\theta(0), \\
& R(x)=x^{\rho} a([x]) \exp \left\{\int_{0}^{x} \frac{\varepsilon([t+1])}{[t+1]} d t\right\},
\end{aligned}
$$

for $x>0$, defining $a(0)$ by $a(0)=1$, say. A glance at (1.4), or a direct verification using the definition of a regularly varying function, shows $R(x)$ is regularly varying with index $\rho$, and $R(n)=\theta(n), n \geqq 0$.

Necessity. Conversely, if $R(x)$ is a regularly varying function on $[0, \infty)$ of index $\rho$ then we have, for $x \geqq B \geqq 0$,

$$
R(x)=x^{\rho} \exp \left\{\eta(x)+\int_{B^{\prime}}^{x} \frac{\varepsilon(t)}{t} d t\right\}
$$

from (1.4), where $\varepsilon(t)$ may be taken as continuous for $x \geqq B, \varepsilon(t) \rightarrow 0$ as $t \rightarrow \infty, \eta(x) \rightarrow c(|c|<\infty)$. For integer $n \geqq 0$, we have $\theta(n)=R(n)$, so for 
integer $n \geqq B$

$$
\theta(n)=n^{\rho} \exp \left\{\eta(n)+\int_{13}^{n} \frac{\varepsilon(t)}{t} d t\right\}
$$

since we are assuming $\theta(n)$ is imbeddable in $R(x)$.

To verify (2.1a) and (2.1b), put $\alpha(n)=n^{\rho} \exp \left\{\int_{B}^{n} \varepsilon(t) / t d t\right\}$ for all sufficiently large $n$; then $\theta(n) \sim K \alpha(n), K$ a positive constant; also

$$
n\left(1-\frac{\alpha(n-1)}{\alpha(n)}\right)=n\left(1-(1-1 / n)^{\rho} \exp \left\{-\int_{n-1}^{n} \frac{\varepsilon(t)}{t} d t\right\}\right) \rightarrow \rho
$$

by using power series expansions, noting that $n \int_{n-1}^{n} \varepsilon(t) / t d t=n\left[\varepsilon\left(\xi_{n}\right) / \xi_{n}\right]$ where $n-1<\xi_{n}<n$ by the mean value theorem $(\varepsilon(t)$ being continuous).

COROLlaRY. If a sequence of positive terms $\{\theta(n)\}, n \geqq 0$, is regularly varying with index $\rho$, then so is the positive function $\theta(x), x \in[0, \infty)$, defined in terms of the sequence by $\theta(x) \equiv \theta([x]), x \geqq 0$.

Proof. Let $R(x), x \in[0, \infty)$, be a regularly varying function of index $\rho$, in which $\{\theta(n)\}$ is imbeddable. Then, for $x>0$,

$$
\begin{aligned}
\theta(x) & =\theta([x])=R([x])=R\left(x-\delta_{x}\right), \quad \text { where } 0 \leqq \delta_{x}<1 ; \\
& =R\left(x\left(1-\left(\delta_{x}(x)\right)\right), \quad \sim R(x), \text { as } x \rightarrow \infty,\right.
\end{aligned}
$$

by the uniform convergence property of regularly varying functions.

\section{REFERENCES}

J. Aczél, Vorlesungen über Funktionalgleichungen und ihre Anwendungen, Lehrbücker und Monographien aus dem Gebiete der exakten Wissenschaften, Mathematische Reihe, Band 25, Birkhäuser Verlag, Basel, 1961; English transl., Math. in Sci. and Engineering, vol. 19, Academic Press, New York, 1966. MR 23 \#A1959; 34 \#8020.

R. Bojanić and E. Seneta, Slowly varying functions and asymptotic relations, J. Math. Anal. Appl. 34 (1971), 302-315. MR 43 \#438.

N. G. de Bruijne, Pairs of slowly oscillating functions occurring in asymptotic problems concerning the Laplace transform, Nieuw. Arch. Wisk. (3) 7 (1959), 20-26. MR 21 \#5847.

L. de Haan, On regular variation and its application to the weak convergence of sample extremes, Mathematical Centre Tracts, 32, Mathematisch Centrum, Amsterdam, 1970. MR 44 \#3370.

I. A. Ibragimov and Ju. V. Linnik, Independent and stationarily connected variables, Nauka, Moscow, 1965; English transl., Noordhoff, Groningen, 1971. MR 34 \#2049.

J. Karamata, Sur un mode de croissance régulière des fonctions, Mathematica (Cluj) 4 (1930), 38-53.

- Sur un mode de croissance régulière. Théorèmes fondamentaux, Bull. Soc. Math. France 61 (1933), 55-62. 
J. P. Kubilius, Probabilistic methods in the theory of numbers, Gos. Izdat. Polit. Naučn. Lit. Litovsk. SSR, Vilna, 1962; English transl., Transl. Math. Monographs, vol. 11, Amer. Math. Soc., Providence, R.I., 1964. MR 26 \#3691; 28 \#3956.

R. Schmidt, Über divergente Folgen und lineare Mittelbildungen, Math. Z. 22 (1925), 89-152.

I. Schur, Zur theorie der Césaroschen und Holderschen Mittelwerte, Math. Z. 31 (1930), 391-407.

Department of Mathematics, Temple University, Philadelphia, Pennsylvania 19122 (Current address of J. Galambos)

Department of Statistics, Princeton University, Princeton, New Jersey 08540

Current address (E. Seneta): Statistics Department, Australian National University, Canberra, Australia 